\title{
Implementation of Computer Aided Diagnosis System for Lung Cancer Detection
}

\author{
Naveed Ejaz, Shazia Javed, and Zeeshan Sajid
}

\begin{abstract}
Computer Aided Diagnosis in radiology assists the radiologists in determining medical abnormalities by providing automated analysis of medical images. The clinical acceptance of Computer Aided Diagnosis systems depends on successful execution of two tasks: segmentation of organ of interest, and identification and classification of abnormalities present on the organ. In this paper we present in detail the implemented Computer Aided Diagnosis process for lung cancer detection, and a novel technique entitled "Contour Detection Method" for identification of lung cancer nodules. We describe the various algorithms employed for each step of the diagnosis process. The implemented algorithms are tested for the Lung Image Database Consortium (LIDC) comprising of CT scan images in DICOM format. The experimentation results for the same dataset reveals that the proposed technique outperforms widely employed Local Density Maximum algorithm by detecting and classifying $7 \%$ of the observed false negatives.
\end{abstract}

Index Terms-Computer aided diagnosis, multi-sliced CT, lung cancer nodules, contour detection method, local density maximum.

\section{INTRODUCTION}

Computer Aided Diagnosis (CAD) is a relatively young technology combining elements of computer vision: an application area of artificial intelligence, and digital image processing. The applications of CAD include identification of cancer in $\operatorname{lung}(\mathrm{s})$, breast(s), and colon, coronary artery disease, heart congenital defect, and nuclear medicine. In radiology CAD implies that the radiologist uses the outcome of computerized analysis of a medical image [1]. The underlying idea is to increase overall sensitivity detection rate through automated identification of suspicious objects, rather than absolute delegation of diagnosis to deployed CAD system. Consequently, the associated advantages are manifold. CAD increases therapy success in case of early disease detection, and may avoid unnecessary biopsies. Further, it reduces error rate in screening situations with massive load of medical images [2].

Manuscript received May 15, 2013; revised July 26, 2013. The test data and domain knowledge for this research was provided by Shaukat Khanum Memorial Hospital, Lahore, and NESCOM Hospital, Islamabad, Pakistan respectively.

N. Ejaz is with Intelligent Media Lab, Department of Digital Contents, Sejong University, Seoul, Korea, on leave from the National University of Computer and Emerging Sciences (NUCES aka FAST-NU), Islamabad, 44000 Pakistan(e-mail: naveed.ejaz@ nu.edu.pk).

S. Javed was with the National University of Computer and Emerging Sciences (NUCES aka FAST-NU), Islamabad, 44000 Pakistan. She is now with the Department of Computer Science, University of Tartu, Tartu, Estonia (e-mail: shazia.javed@ut.ee).

Z. Sajid was with the National University of Computer and Emerging Sciences (NUCES aka FAST-NU), Islamabad, 44000 Pakistan. He is now with EssClaims, Cambridge, UK (e-mail: zeeshan@essclaims.com).
Among the aforementioned cancers, lung cancer is categorized as the one with the smallest survival rate after diagnosis, and with gradually increasing mortality rate per annum [3]. According to American Cancer Society there exists a survival rate of $47 \%$, if lung cancer is detected in early stage [4].

In $\mathrm{CAD}$, the process of detecting lung cancer involves two tasks: identification and classification. The identification task pertains to finding lung cancer nodules in medical images. Lung cancer nodule, also referred as solitary pulmonary nodule or coin lesion, is visible as a mass in lung spanning a diameter smaller than 3 centimeter. The classification task comprises of two stages. First of all, candidate nodules are classified as nodules or non-nodules. Second, the determined nodules are further classified as benign or malignant. Lung nodules are usually an incidental finding in up to $1 \%$ of the CT scan images with $20 \%$ cases of malignant cancer and 10-20\% diagnosis percentage for lung cancer patients [5].

Considering the relatively high probability of curing early lung cancer, this research effort focuses on identification and classification of lung cancer nodules in CT scan images. The following section describes in detail our implementation of the $\mathrm{CAD}$ process for lung cancer detection. It also introduces a novel identification technique "Contour Detection Method", and presents comprehensive comparative analysis of the proposed detection algorithm and widely employed Local Density Maximum algorithm. The paper concludes with a summary of our research effort and future possibilities.

\section{The COMPuter Aided Diagnosis Process}

The CAD process for lung cancer nodule detection consists of following phases:

\section{A. Image Acquisition}

Image acquisition is a difficult job as there are no public databases of CT scan images, and hospitals are reluctant to handover the medical data. The medical data is usually in DICOM format, which is the standard for storage and transfer of medical images [6]. The DICOM format can be exported to bitmap format. Our implementation benefits from the bitmap conversion of acquired DICOM CT scan images.

\section{B. Image Preprocessing}

For efficient lung nodule detection and classification, it is necessary to extract the area of interest i.e. lungs, and discard the irrelevant information like fat, bones, bed, background from the CT scan image.

In literature two types of techniques are suggested for extraction of lungs: rule-based reasoning and pixel classification [7]. Rule-based reasoning techniques are a 
sequence of steps, tests, and rules. These include techniques like Local Thresholding, Region Growing, Edge Detection, and Morphological operations. In pixel classification, each pixel from the image is classified into an anatomical class (usually lung or background, but in some cases more classes like heart, mediastinum, and diaphragm are used) [3], [7].

Our implemented technique falls under the rule-based reasoning category. In our approach, the basis of lung segmentation relies on finding a threshold in the density histogram of CT scan images. This approach was suggested in [8] and comprises of following steps:

\section{1) Get gray scale image}

The acquired image is converted into 16 bit gray scale image. This helps in reducing the total number of distinct pixel values. Our implementation relies on the relation (1) used by GNU Image Manipulation Program.

GrayPixel $=0.3 \times$ RedPixel $+0.59 \times$ GreenPixel $+0.11 \times$ BluePixel

Fig. 1 shows the actual image and its corresponding gray scale image.
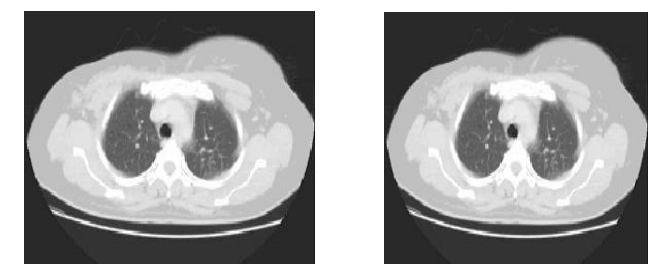

Fig. 1. Actual image (left) and its gray scale counterpart (right).

\section{2) Density histogram and threshold selection}

A histogram shows the distribution of data values. It bins the pixels into $n$ equally spaced containers where $n$ is the number of distinct pixels in the image. For each bin of distinct pixel values, the number of pixels lying in that bin are stored and plotted.

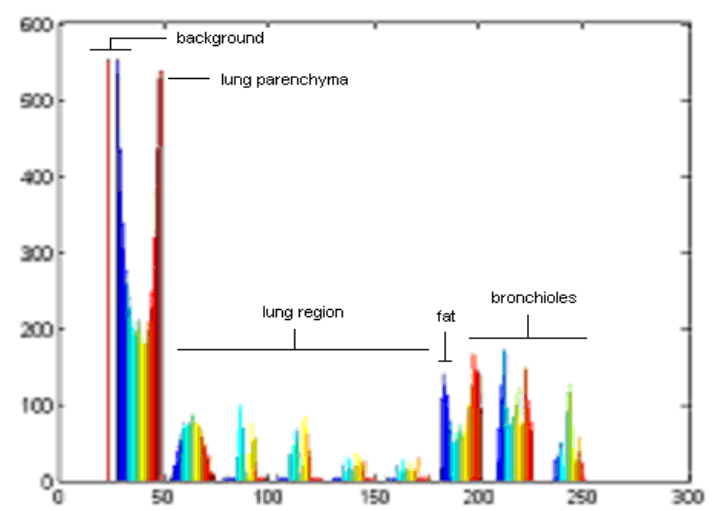

Fig. 2. Histogram of a given CT scan image.

Experimentation shows that usually there are four main peaks on the histogram of a give CT scan image. The peaks (from left to right in Fig. 2) represent background outside the scanned body, lung parenchyma, fat, and muscles, respectively. Human bones have higher density than those of muscles but their corresponding pixels are too few in number to form a peak. A long, flat, and low valley between the peaks of the lung parenchyma (second peak from the left) and the fat (third peak from the left) on the histogram indicates the separation of the lung parenchyma from the soft tissues (fat and muscles) and bones. A threshold value thus lying in the lung parenchyma-fat valley can be chosen once the high peaks have been determined automatically.

This threshold is used to separate the lung parenchyma from the other anatomic structures on the CT scan images. In our implementation, the threshold is chosen experimentally. The results indicate that the pixels with gray scale value larger than 150 constitutes the lung region. Consequently, pixels with gray scale value less than the threshold value are turned white, whereas pixels value greater than the threshold are changed to black. This gives us the Thresholded Image, in Fig. 3, which is a binary image.

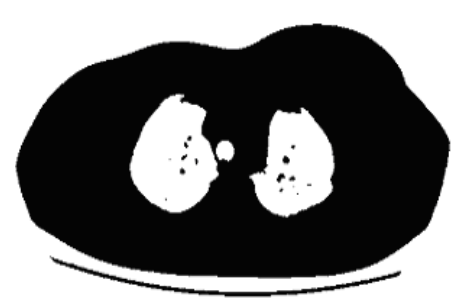

Fig. 3. Thresholded CT scan image.

\section{3) Initial lung mask}

The problem with the thresholded images is that due to their low densities both the lung parenchyma and background will be classified as lung on the resultant binary images. It is clear from the CT scan image that the lung parenchyma is usually completely isolated from the background by the chest wall. It can be readily determined by labeling the connected components i. e. grouping geometrically connected white pixels and assigning an identical number to the pixels in each individual group. These connected components are found by using the 4-point connectivity algorithm [9]. Each distinct connected group is assigned a unique integer value.

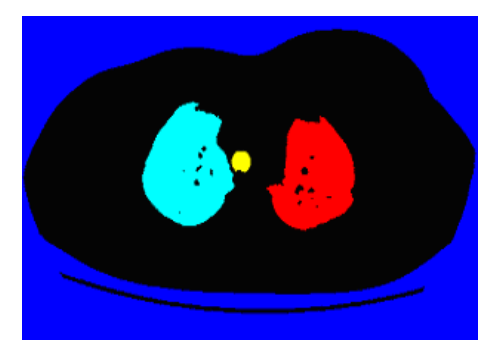

Fig. 4. Connected components in a CT scan image.

In Fig. 4 each connected component is shown in different color. We select the largest component that does not touch any margin of the images, and in this way we alos manage to separate background as is shown in Fig. 5.

\section{4) Complete lung mask}

As the apparent density of vessels and bronchial walls in the lung is different, structures with higher densities including some higher density nodules could get grouped into soft tissues and bones. This leads to an incomplete extraction of the lung mask as is shown in Fig. 5. Thus the mask is not complete and has holes.

In order to obtain a complete, hollow-free lung mask, morphological closing [10] is applied. Disk shape of the structural element is chosen for the morphological operator and the disk size is experimentally determined. After this step 
the holes are filled and complete lung mask is obtained as shown in Fig. 6.

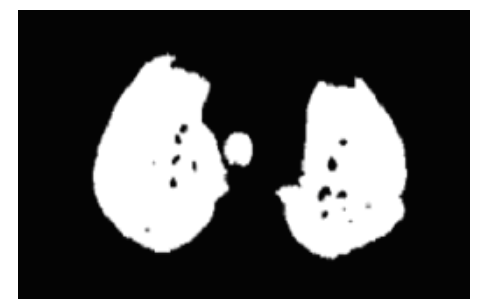

Fig. 5. Initial lung mask for extracting area of interest.

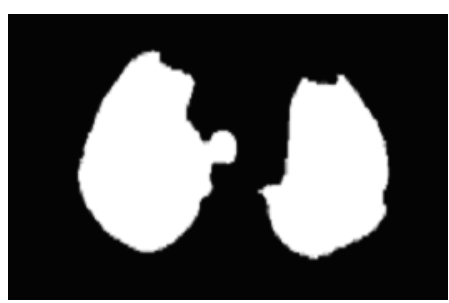

Fig. 6. Complete lung mask for extracting area of interest.

\section{5) Extraction of lungs}

With the availability of Complete Lung Mask, the lungs can be readily extracted from the original CT scan images. For this, the Complete Lung Mask is compared with the actual CT scan image. The pixels from the actual image corresponding to the white pixels in the masked image are retained. Fig. 7 shows the lungs extracted from the actual image.

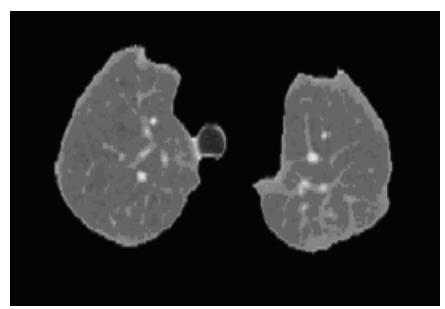

Fig. 7. Extracted lungs from the actual CT scan image.

\section{Nodule Detection}

Nodules appear as relatively low-contrast white circular objects within the lung fields. The detection of such cancerous nodules is not trivial because of the existence of other similar and misleading anatomical structures such as blood vessels and airways.

All nodule detection techniques proposed in the literature can be classified as either density based or model based techniques. The density based techniques are based on multiple thresholding, region growing and clustering, whereas the model based techniques focuses on the peculiar shape of nodules which is different from other anatomical structures.

\section{1) Local density maximum}

Local Density Maximum (LDM) [11] is a density-based technique which exploits the fact that in CT images blood vessels, bronchial walls, and nodules have density values higher than those of the lung parenchyma. Therefore, this technique focuses on finding objects that have high density in their neighborhood.

Before presenting the algorithm, we define a plateau as a region where two local maxima are present side by side in close neighborhood. Further, we enlist five parameters which are used later:

1) Threshold Start Value specifies the density value from where thresholding begins.

2) Threshold Step Value specifies the step by which threshold value is decreased at each step.

3) The Threshold Stop Value is the density value at which the algorithm is stopped.

4) Minimum density of local maximum

5) Ratio value specifying the change of object's volume to its surrounding box's volume.

All values are determined experimentally. Initially the maximum density of the CT scan image is selected as the Threshold Start Value. All objects above this threshold value are found. This threshold is then decreased successively by Threshold Stop Value that is fixed in the first step. The CT scan image is converted into Black and White image based on the threshold value. Following to this, the connected components are determined in this Black and White image. This step is repeated by decrementing threshold value by Threshold Step Value. The objects at current threshold level may have some objects detected at previous threshold level. For a given object $i$ at current threshold level, there exists $m$ objects detected at the previous level such that object $i$ contains all of these $m$ objects within it. This step is repeated for all current nodules. The zero value for $m$ means the newly detected object contains no object of previous level within it. This means that this object is a new object and added into the stack of currently detected nodules. The $m$ value of 1 means that the current object contains only one object from the previous level. To tackle this we define Bounding Box of an object as the smallest rectangle containing the object. We also define

1) $d x$ is the difference between the highest $\mathrm{x}$-coordinate in the object and the lowest $\mathrm{x}$-coordinate in the object.

2) $d y$ is the difference between the highest y-coordinate in the object and the lowest $y$-coordinate in the object.

3) $d$ is defined as the square of the maximum of $d x$ and $d y$ value.

4) $R 1$ is the number of pixels in current object divided by $d$.

5) $R 2$ is the number of pixels in previous object divided by $d$.

6) $R$ is the ratio of $R 1$ to $R 2$.

If the value of $R$ is less than the Ratio defined in the first step, then this means that $R$ drops drastically. In this scenario, the current object will be interpreted as a plateau and the previous object replaces new object. If either $R$ is greater or the previous object does not meet criteria of being local maxima, the current object replaces the previous object. If the value of $m$ is greater than 1 , it means that the current object contains more than one previous object. All of those previous objects which satisfy the criteria of being local maxima as indicated in the first step are recognized as local maxima while the current object is recognized as plateau. If only one of the previous object meets the criteria of first step then it is treated as in the second case (i.e. $m=1$ ). If none of the previous object meets the criteria it is treated as the first case $(m=0)$.

The process of searching for local maxima terminates when the threshold reaches the minimal density value as 
specified in the first step or a predefined density value which can be obtained by statistical analysis of lung parenchyma.

To illustrate the idea further, let $P 1, P 2, P 3$ and $P 4$ be the objects detected at the previous threshold value and $C 1, C 2$ and $C 3$ be the objects detected at the current threshold value. Table I lists the points included in these object. The first current object $C 1$ does not overlap with any of the previous object so in this case $m=0 . C 2$ overlaps with only one previous object i.e. $P 1$ so in this case $m=1$. Finally $C 3$ overlaps with $P 2$ and $P 3$ and so for this object we follow case of $m>1$.

TABLE I: PREVIOUS AND CURRENT OBJECTS IN LDM

\begin{tabular}{lllllll}
\hline \hline$P 1$ & $P 2$ & $P 3$ & $P 4$ & $C 1$ & $C 2$ & $C 3$ \\
\hline$(1,1)$ & $(25,26)$ & $(50,51)$ & $(51,53)$ & $(60,61)$ & $(1,1)$ & $(50,51)$ \\
$(1,2)$ & $(25,27)$ & $(51,52)$ & $(51,54)$ & $(61,62)$ & $(1,2)$ & $(51,52)$ \\
$(1,3)$ & $(26,26)$ & & & $(62,63)$ & $(1,3)$ & $(51,53)$ \\
$(1,4)$ & $(26,27)$ & & & & $(1,4)$ & $(51,54)$ \\
& & & & & $(1,5)$ & $(52,53)$ \\
& & & & & $(2,6)$ & $(52,54)$ \\
\hline \hline
\end{tabular}

\section{2) Contour detection method}

We propose a new model-based technique for the detection of candidate nodules. Before we present the method itself, we provide two definitions which are employed in the description later:

1) Closed Path begins and ends at the same point.

2) Simple Closed Path is a closed path that does not touch or intersect itself. For example, a circle is a simple closed path while an 8 -shaped curve is not. A simple closed path is also called a Contour.

It can be observed readily by looking at the CT scan image of a lung that all candidate nodules are found in the form of simple closed paths. Therefore, the problem of finding nodules is reduced to highlighting all the contours within lung parenchyma.

A contour graph displays isolines of matrix $Z$ where matrix $Z$ is a two-dimensional image matrix. $Z$ is interpreted as heights with respect to the $x-y$ plane. The value of $x$ is chosen between 1 and $m$, and the value of $y$ is chosen between 1 and $n$, where $m$ and $n$ represents rows and columns of the image matrix respectively. In image processing, the boundary of a contour is decided by abrupt change in the gray value (same definition as that of an edge).

The proposed Contour Detection Method (CDM) starts by considering whole of the image as one contour. Later whenever an abrupt change is found in the image we say that a contour is anticipated. The 8-neighbours [12] of this abrupt change are checked recursively until the neighbor changes abruptly. This yields a contour and its boundary is given a specific color. All of the contours are highlighted in the similar way.

Our implementation further modifies the algorithm to detect contour(s) after thresholding. As in the CT scan images, blood vessels, bronchial walls, and nodules have density values higher than those of the lung parenchyma. Lower density values are eliminated by means of thresholding. The modified technique is named "Contour Detection with Background Elimination". This so-called background elimination removes unnecessary overhead of finding contours in the low-density region in which we are not interested. Fig. 8 shows the highlighted nodules in the lung region.

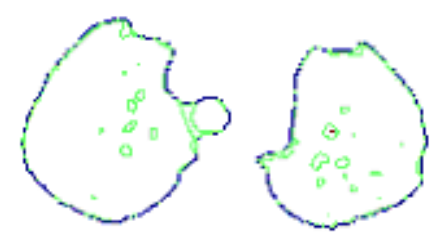

Fig. 8. Contours highlighted on the processed CT scan image.

\section{3) Comparison of LDM and CDM}

The Contour Detection Method is simple, easy to understand and fast as compared to Local Density Maximum. However, it tend to miss out the nodules that are smaller in size as they may comprise of one or two pixels and thus does not come under the definition of contour. This is a serious drawback of the proposed method. However, such nodules tend to be rare. Further, this drawback can be eliminated by slightly modifying the definition of a contour. Let $n$ be the number of pixels in an object then if $n \leqslant 2$ then the object is a contour otherwise the regular definition of contour (already given) is applied for lung nodule detection. This slight modification solves the problem of small nodules in the proposed method.

We designed a few test cases for the comparison of LDM and CDM. The proposed method (without modification to the definition of contour) shows promising results and catches $95 \%$ of the nodules detected by LDM. The nodules missed by CDM are likely to be identified by the modification suggested above, which remains to be tested. Table II lists the central pixel location of nodules from a CT scan image, and identification status for both methods.

TABLE II: COMPARISON BETWEEN LDM AND CDM

\begin{tabular}{lcc}
\hline Lung Nodule Center & Detected by LDM & Detected by CDM \\
\hline$(108,154)$ & Yes & Yes \\
$(109,170)$ & Yes & Yes \\
$(112,182)$ & Yes & Yes \\
$(94,191)$ & Yes & No \\
$(88,197)$ & Yes & Yes \\
$(97,220)$ & Yes & No \\
$(110,109)$ & Yes & Yes \\
$(128,186)$ & Yes & No \\
$(86,206)$ & Yes & Yes \\
$(108,226)$ & Yes & \\
\hline \hline
\end{tabular}

\section{Feature Extraction}

Next step to nodule detection is differentiation between nodules and non-nodules (vessels, benign nodules, airways etc.). Further, the true positives need to be classified as either benign or malignant. For this purpose, we need to extract certain properties, measures, or features from the CT scan image.

We have determined following features as essential to the classification process:

1) Number of Pixels (Area of Object) is the simple count of the number of pixels present in the candidate nodule. This feature incorporates the size factor of the nodules.

2) Average Intensity is calculated by summing up 
intensities of all of the nodule's pixels and then dividing the sum by the number of pixels. Although intensity of various anatomical structures is very close to that of nodules, yet a small difference can be exploited for classification.

3) Ratio of object's pixels to its Bounding Box (where a bounding box is the smallest rectangle which encloses the nodules completely within itself) covers the propensity of structures towards rectangular shape. Since nodules are not rectangular so this value must be very small as compared to that of non-nodules.

4) Eccentricity is the ratio of the distance between the foci of the ellipse and its major axis length. The eccentricity of the ellipse which encompasses a nodule is measured. The eccentricity can be interpreted as the fraction of the distance along the semi major axes at which the focus lies. It is given by $e=c / a$ where $c$ is the distance from the center of the conic section to the focus. The value of eccentricity lies between 0 and 1 both inclusive. If the value determined is 0 , the object is a circle where as if the value is 1 , the object is a straight line. Again this feature covers the shape aspect of the nodules. Nodules have a general propensity towards spherical shape so eccentricity of nodules must be close to 0 .

5) Solidity of a nodule is determined by the proportion of the pixels in the convex hull that are also in the region. A set $C$ is convex if the line segment between any two points in $C$ lies in $C$. The Convex Hull is the smallest convex polygon that can contain the region. Again this feature encompasses the shape aspect of the nodule.

6) Major Axis Length is the length (in pixels) of the major axis of the ellipse which encompasses region. This encompasses within itself the spherical shape of the nodule.

7) Minor Axis Length is the length (in pixels) of the minor axis of the ellipse which encompasses region. This encompasses within itself the spherical shape of the nodule.

\section{E. Nodule Classification}

Based on the features identified in the previous step, the classification step tries to minimize the number of false positive, and to differentiate between the nodules and the non-nodules. For classification purposes we employ the PUBLIC algorithm [13]. The goal of PUBLIC algorithm is to induce a model or description for each class in terms of the attributes. A decision tree is built whose internal node has a test involving an attribute, and an outgoing branch for each possible outcome. Each leaf has an associated class. In order to classify new records using a decision tree, beginning with the root node, successive internal nodes are visited until a leaf is reached. At each internal node, the test for the node is applied to the record. The outcome of the test at an internal node determines the branch traversed, and the next node visited. The class for the record is simply the class of the final leaf node. Thus, the conjunction of all the conditions for the branches from the root to a leaf constitutes one of the conditions for the class associated with the leaf.

For our implementation, we determined the values of all the features for the nodules and non-nodules identified by the radiologist (see Table III). The measured values served as the small data set for the generation of rules using the PUBLIC algorithm [13]. The generated rules assisted in classification of candidate nodule.

TABLE III: FEATURE VALUE FOR SAMPLE NODULE AND NON-NODULE

\begin{tabular}{lcc}
\hline \hline Feature Type & Nodule & Non-Nodule \\
\hline Area of Object & 74 & 34 \\
Average Intensity & 122 & 200 \\
Ratio of Object Pixels to its Bounding Box & 61 & 53 \\
Eccentricity (x100) & 60 & 9 \\
Solidity (x100) & 99 & 100 \\
Major Axis Length (x100) & 11 & 7 \\
Minor Axis Length (x100) & 9 & 7 \\
\hline \hline
\end{tabular}

Since the classification results improve with the size of data set, we further tested our proposed implementation for CT scan images from the Lung Image Database Consortium (LCID). For this collection, our proposed implementation exceeds the performance of LDM by detecting and classifying false negatives in $7 \%$ of the images, thereby acknowledging the completeness and generality of rules for completely discerning the nodules from the non-nodules. We conclude that provided the data set is sufficiently large, the proposed algorithm as well as feature set will definitely work with sufficient accuracy.

\section{SUMmary AND Future Directions}

In this paper we present the CAD process for detecting lung cancer in CT scan images, and its implementation. One of the apprehensions of using CT for lung cancer screening is the large number of images that need to be interpreted by the radiologist. In such a scenario, employing a CAD system may improve cancer detection rate and reduce oversight errors. Considering the associated advantages, we present in detail the implementation of CAD process for lung cancer detection.

First, a rule-based approach is selected from the literature and implemented for image pre-processing. This approach is simple and easy to understand as the usage of histogram and density analysis is well-established in the field of Image Processing. Second, Local Density Maximum algorithm is implemented for candidate nodule detection. This algorithm exploits the density variations between various parts of lung parenchyma. A new technique "Contour Detection Method" is also presented for this purpose. A comparison of these two techniques is presented which shows that the proposed contour method tends to detect $95 \%$ of the nodules that are being detected by LDM. The modified version of Contour Method is also suggested which is likely to overcome the drawbacks in the proposed method. The modification remains to be tested. We also identify seven classification features, and accumulate associated dataset for nodules and non-nodules. We have proposed a new feature Solidity which has a profound effect in deciding the shape of the nodule. The implemented system uses the feature dataset for generating classification rules, which in turn are used for classifying candidate nodules as nodule or non-nodules.

The performance of proposed diagnosis process improves as a large dataset is employed. For the images from LIDC collection, our approach outperforms LDM by identifying 
and classifying $7 \%$ of the observed false negatives. Therefore, we conclude that the presented CAD process is expected to perform with high accuracy given the availability of large dataset. Also, the presented techniques are expected to be of much help for further research in this area. The implementation of error free CAD system, however, requires availability of sufficiently large dataset and arduous effort from both researchers and radiologists.

Our future efforts concentrate on thorough testing of the presented CAD process, and verification of the modified Contour Detection Method. We also intend to perform extensive literature survey and acquire domain knowledge essential to probable application of proposed approach to 3D constructions of CT scan images, and automation of nodule classification as either benign or malignant.

\section{ACKNOWLEDGMENT}

We are thankful to Dr. Najum-ud-din, Head of Radiology department in Shaukat Khanam Hospital, Lahore, for his help and support in data acquisition, and Dr. Shahid Manzoor, Radiologist, Nescom Hospital Islamabad, for his help and support in Nodule classification.

\section{REFERENCES}

[1] L. G. Maryellen, "Computer aided diagnosis in radiology," Acad Radiol, vol. 9, pp. 1-3, 2002.

[2] J. M. Goo et al., "Automated lung nodule detection at low-Dose CT: Preliminary experience," Korean Journal of Radiology, vol. 4, December 2003.

[3] N. Vittitoe, R. Vargas-Voracek, and C. Floyd Jr., "Markov random field modeling in posteroanterior chest radiograph segmentation," Med. Phys., vol. 26, pp. 1670-1677, 1999.

[4] V. K. Soni. (May 2009). Lung Cancer Treatments and Survival Rate. Steady Health. [Online]. Available: http://goo.gl/1iQnQ.

[5] K. Alzahouri, M. Velten, P. Arveux, M. C. Woronoff-Lemsi, D. Jolly, and F. Guillemin, "Management of SPN in france. Pathways for definitive diagnosis of solitary pulmonary nodule: A multicentre study in 18 French districts," BMC Cancer, vol. 8, 2008.

[6] Digital Imaging and Communications in Medicine (DICOM) Part 1: Introduction and Overview, National Electrical Manufacturers Association, 2006, pp. 11.

[7] M. McNitt-Gray, H. Huang, and J. Sayre, "Feature selection in the pattern classification problem of digital chest radiograph segmentation," IEEE Trans. Med. Imag., vol. 14, pp. 537-547, June 1995.

[8] C. I. Henschke, D. I. McCauley, and D. F. Yankelevitz, "Early lung cancer action project: Overall design and findings from baseline screening," Lancet, vol. 354, no. 9173, pp. 99-105, 1999.
[9] A. Kanevsky, and V. Ramachandran, "Improved algorithms for graph four-connectivity," in Proc. 28th Ann. IEEE Symp. on Foundations of Comp. Sci., 1987, pp. 252-259.

[10] E. R. Doughtery and R. A. Lotufo, Hands-on Morphological Image Processing, 1st ed., Chichester, WA: SPIE, 2003, ch. 2-3, pp. 25-64.

[11] B. Zhao et al., "Automatic detection of small lung nodules on CT utilizing a local density maximum algorithm," Journal of Applied Clinical Medical Physics, vol. 4, no. 3, 2003.

[12] T. Cootes, C. Taylor, D. Cooper, and J. Graham, "Active shape models-Their training and application," Comput. Vis. Image Understanding, vol. 61, no. 1, pp. 38-59, 1995.

[13] R. Rastogi and K. Shim, "PUBLIC: A decision tree classifier that integrates building and pruning," $V L D B$, pp. 404-415, 1998.

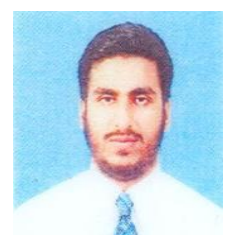

Naveed Ejaz graduated with bachelor of science in computer science from National University of Computer and Emerging Sciences (NUCES aka FAST-NU), Islamabad, Pakistan in 2005. He completed his Master of Science in computer software engineering from National University of Science and Technology (NUST), Islamabad, Pakistan in 2007. He defended his doctoral thesis in image processing at Sejong University, Seoul, Korea in 2013.

$\mathrm{He}$ is currently working as a research assistant at Intelligent Media Lab, Sejong University, Korea. His past affiliations include working as Lecturer and later Assistant Professor with FAST-NU. His research efforts focus on topics from computer vision, specifically image processing.

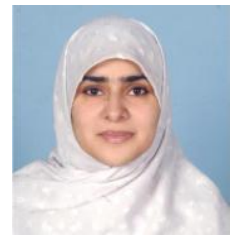

Shazia Javed graduated with bachelor of science in computer science from National University of Computer and Emerging Sciences (NUCES aka FAST-NU), Islamabad, Pakistan in 2005, and graduated cum laude from University of Tartu, Tartu, Estonia with Masters of Science in Engineering (software engineering) in 2012.

She is currently working as a research engineer with Scribeneur in Tartu, Estonia. Her previous work experience includes the role of Software Engineer and Business / Software Analyst with InterActive Covergence, Teaching Assistant with University of Tartu and FAST-NU, and Lecturer BS(CS) with Islamabad Model College for Girls St. 25 F-6/2, Islamabad, Pakistan. Her research interests include Machine Learning and Computer Vision.

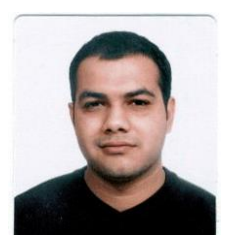

Zeeshan Sajid graduated with bachelor of science in computer science from National University of Computer and Emerging Sciences (NUCES aka FAST-NU), Islamabad, Pakistan in 2005.

$\mathrm{He}$ is currently working as a director with EssClaims, Cambridge, United Kingdom. He is also serving as the Chief Technology Officer of Xeeonix Technologies. His previous work experience includes the role of Software Developer with AdvancedVoIP and Software Engineer with Media Routes Inc. 